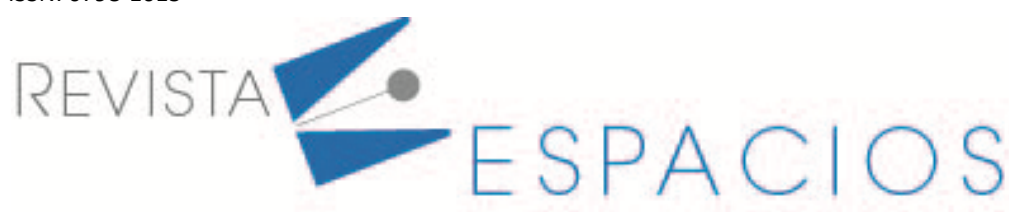

Vol. 42 (12) $2021 \cdot$ Art. 9

\title{
La función identitaria en la comunicación de proximidad. El caso de la televisión regional de Castilla-La Mancha (España)
}

The identity function in proximity communication. The case of regional television in CastillaLa Mancha

GONZÁLEZ, Gabriel ${ }^{1}$

\begin{abstract}
Resumen
El objetivo del presente trabajo es contribuir a clarificar si el modelo de televisión pública ha sido eficaz para cumplir la función identitaria en una Comunidad Autónoma española de las llamadas no históricas. Para analizar la programación, se han seleccionado una serie de indicadores que puedan aplicarse sobre los programas emitidos, tratando de establecer si la susodicha programación ha estado planteada como una contribución a la promoción y difusión de los aspectos identitarios de la región.

Palabras clave: televisión, identidad, medios de comunicación, cultura

Abstract

The objective is to help to clarify if the public television model presently used has effectively fulfilled its function as regards establishing a separate regional identity in a region of Spain. In order to analyze the programming, a series of indicators were selected that were then applied to the programs broadcast by the channel, trying, in this way, to establish if the aforementioned programming has contributed to the promotion and diffusion of the identity aspects of the region.

Key words: television, identity, mass media, culture
\end{abstract}

\section{Introducción}

En un mundo globalizado, y aunque pueda parecer paradójico, lo local ha cobrado importancia. El difuminado de las identidades en un conjunto mundial, o al menos occidental, ha dado por resultado una respuesta en forma de promoción del arraigo regional. En este sentido, en España, y en relación a los medios de comunicación, la aparición de las televisoras ha seguido dos velocidades. Así, mientras en las comunidades con mayor población este tipo de televisoras se generaron durante los ochenta, la mayor parte de las comunidades con menor cantidad de ciudadanos no tuvieron televisiones regionales hasta los años dos mil, caso del canal de televisión que se va a tratar en este trabajo, Castilla-La Mancha Media (CMM), que vio la luz en 2001. En este sentido, uno de los conceptos que han necesitado de definición y reflexión ha sido el concepto de identidad cultural, puesto que para poder determinar hasta qué punto la televisión regional castellanomanchega ha tenido (y tiene) influencia en la generación y desarrollo de la identidad cultural regional, será necesario tener claras las diferentes definiciones que la bibliografía le ha otorgado.

\footnotetext{
${ }^{1}$ Profesor Asociado. Departamento de Humanidades. Universidad Rey Juan Carlos. España. gabriel.gonzalez@urjc.es
} 
Otro apartado que ha necesitado de una intensa revisión bibliográfica es el referente a los medios de proximidad. En este sentido, existen diversos trabajos que han tratado estos aspectos, los cuales han servido, sin duda, como base sobre la que construir la investigación que se ha llevado a cabo y que forma parte de un estudio mucho más extenso sobre la construcción de la identidad regional a través de la comunicación de proximidad. Por ello, lo que se propone con este artículo es contribuir a clarificar si el modelo de programación de la televisión pública de Castilla-La Mancha es un vehículo eficaz y de utilidad para la construcción de la identidad regional en una Comunidad Autónoma de las llamadas no históricas, ahondando en un mejor conocimiento del único medio televisivo de carácter regional que opera en la misma. Para ello se ha realizado un análisis exhaustivo de la programación de la cadena, y se han seleccionado una serie de indicadores para valorar si dicha programación se ha planteado para la promoción y la difusión de la identidad cultural de la región.

\subsection{Identidad cultural}

Desde la década de los noventa, como así se percataba Hall (1996), se ha registrado una explosión discursiva en torno al concepto de identidad. Un aspecto que, no obstante, no debe sorprender, puesto que, como el mismo autor señalaba, los debates sobre la identidad deben situarse dentro de aquellos acontecimientos y prácticas históricamente específicos que han perturbado el carácter relativamente estable de las poblaciones y culturas.

En este sentido, se halla generalmente aceptado que es a partir de los ochenta, y en especial con la entrada de los años dos mil, cuando las distancias y las comunicaciones ven su necesidad de tiempo diluidas a mínimos históricos. Las fronteras (físicas y mentales, y por ende también culturales) se difuminan hasta el punto de confluir como nunca antes, haciéndose cada vez más complicada su diferenciación y especificidad. Motivo por el cual, el estudio de las identidades parece intensificarse, pues resulta un concepto que incrementa su ya de por sí complejo significado. $Y$ es que, al efecto de la globalización, el cual tiene como una de sus principales características la neutralización de las diferencias entre culturas o, si se prefiere, la homogeneización cultural, aparece un efecto de reacción mediante la promoción de lo propio, la recuperación de las propias características y particularidades, en resumen, la recuperación de la identidad (Samour, 2013). A este respecto, Hall (1996) señalaba que las identidades se refieren tanto a la invención de la tradición como a la tradición misma, que nos obligan a leerlo no como una reiteración interminable, ni tampoco como el llamado retorno a las raíces, sino como la llegada a los términos con nuestras "rutas". Surgen de la narrativa del yo, pero la naturaleza necesariamente ficticia de este proceso de ninguna manera mina su efectividad discursiva, material o política, incluso si la pertenencia a través de la cual surgen las identidades está, en parte, en lo imaginario (así como en lo simbólico) y, por lo tanto, siempre, en parte construida en la fantasía. De esta forma, se debe de observar que las identidades se construyen dentro, no fuera, del discurso, por lo que se necesita que se entiendan como elementos que se producen en sitios históricos e institucionales específicos, dentro de formaciones y prácticas discursivas específicas, mediante estrategias enunciativas específicas. Además, emergen dentro del juego de modalidades específicas de poder y, por lo tanto, son más el producto de la diferencia y la exclusión que el signo de una unidad idéntica, constituida naturalmente una "identidad" en su significado tradicional (Hall, 1996).

No obstante, siguiendo el discurso de Giménez (2005, p. 1), al concepto de identidad va ligado, indisolublemente, el concepto de cultura, especialmente si se realiza una aproximación a ellos desde la perspectiva de la antropología y la sociología. Como bien dice el autor, y sin diferir con lo mencionado anteriormente, la identidad consiste en la apropiación de una serie de repertorios culturales que se observan en un entorno social concreto. Especialmente si, tal y como se viene insistiendo, una de las funciones principales del sentimiento de identidad es conseguir una diferenciación entre el nosotros y el ellos, "y no se ve de qué otra manera podríamos diferenciarnos de los demás si no es a través de una constelación de rasgos culturales distintivos".

El término cultura, como ocurre con el de identidades, también destaca por su complejidad y heterogeneidad. Cabe resaltar que, desde Clifford Geertz en los sesenta, la concepción de cultura ha pasado de concebirse como 
unos modelos de comportamientos determinados, a entenderse como a una serie de pautas de significados (Giménez, 2005). Es decir, de nuevo, como ocurría con las identidades, es el simbolismo el factor determinante. Esto mismo señalaba Wallerstein (1989), entendiendo que, mediante la cultura, los diferentes grupos, conseguían representar una serie de características que no eran compartidas (o no completamente) por el resto.

La conjunción de cultura e identidades deriva en la aparición de un tercer elemento, los actores sociales. De nuevo aparecen un sinfín de definiciones para este concepto, pero para el presente estudio trabajaremos sobre la enunciación de Touraine (1987), quien los entiende como un sujeto colectivo que se estructura en base a una conciencia identitaria propia, y que porta y posee una serie de valores y recursos que permiten actuar y defender los intereses de los miembros que la componen y representan.

Otro aspecto a tener en cuenta al hablar de identidad cultural es su carácter histórico, en el sentido en que en su composición se hallan una serie de aspectos que han sido heredados. Aspectos como la lengua, los ritos y tradiciones, las creencias y los sistemas de valores, y, en definitiva, los comportamientos colectivos, son ejemplos de características inmateriales que son fruto de una convivencia colectiva y más o menos extendida en el tiempo (González, 2018). Para muchos, la globalización cultural, el desarrollo de las Tic's, los medios de comunicación, Internet, entre otros factores, están constantemente "hostigando" a la cultura y la identidad, facilitando todavía más el vertiginoso intercambio cultural y, frente a esta situación, no se puede hacer nada, el propio desarrollo actual lo evidencia. Por lo tanto, el desarrollo se impone y si existen elementos que suman, enriquecen, pues se comparten y se valora positivamente la globalización cultural y, por el contrario, si no es así, se debe amparar el desarrollo autóctono, los valores, la historia, en definitiva, la identidad (Álvarez, 2016).

Todo ello unido, cultura, identidades y actores sociales, da lugar a un elemento de enorme importancia en cuanto a la vertebración y desarrollo de un territorio se refiere. Así lo resalta Molano (2007, p. 74), indicando que "muchos pueblos y lugares en Europa y en América Latina han apostado por una revalorización de lo cultural, de lo identitario (recreando incluso nuevas identidades culturales) y patrimonial como eje de su propio desarrollo". Y esto lleva, cerrando el círculo, al aspecto que se trataba al inicio de este apartado, la reacción de recuperación y exaltación de las identidades regionales frente a la amenaza disolvente y globalizadora que supone la globalización. Esta ha barrido como un tsunami las diversas culturas del mundo, destruyendo localidades estables, desplazando a los pueblos, trayendo una homogeneización de la experiencia cultural impulsada por el mercado, eliminando así las diferencias entre las culturas. Aunque se ha considerado que la globalización implica un proceso general de pérdida de diversidad cultural, algunos, por supuesto, mejoraron y otros empeoraron con este proceso. Mientras que esas culturas de la corriente principal del flujo del capitalismo, las occidentales, y de forma especialmente preponderante la emanada por los Estados Unidos, desarrollaron una versión estandarizada de sus culturas para, de una forma más o menos consciente y deliberada, exportarlas a todo el mundo; han sido las culturas "más débiles" las que se han visto más amenazadas. Aunque lo cierto es que las identidades culturales están en peligro en todas partes con la depredación de la globalización (Tomlison, 1999).

No obstante, tal y como señala Castells (2003, p. 24), ante dicha amenaza han aparecido "vigorosas expresiones de identidad colectiva que desafían la globalización y el cosmopolitismo en nombre de la singularidad cultural y del control de la gente sobre sus vidas y entornos". Lejos de ser la frágil flor que pisotea la globalización, la identidad es vista por Castells como el poder emergente de la cultura local que ofrece resistencia (aunque multiforme, desorganizada y a veces políticamente reaccionaria) a la fuerza centrífuga de la globalización capitalista. El impacto de la globalización se convierte así en una cuestión de interacción de un impulso institucional-tecnológico hacia la globalidad con fuerzas "localizadoras" contrapuestas. El impulso hacia la "globalidad" combina una lógica de expansión capitalista con el rápido desarrollo de medios de comunicación y tecnologías de comunicación desterritorializadoras. Pero a este impulso se oponen diversos procesos y prácticas 
que expresan diferentes órdenes de 'localidad'. Y es precisamente este impulso local, regional si se prefiere, el que se está analizando en el presente trabajo.

\subsection{Información de proximidad}

El origen del periodismo, tal y como resaltan López y Maciá (2007), hay que buscarlo en la denominada información de proximidad, puesto que el interés del público, por regla general, siempre se centra en aquello que ocurre en el entorno más inmediato. Una afirmación que aún puede ser comprobada incluso a pesar del grado de globalización cuasi-absoluto en que se hayan la mayor parte de las sociedades hoy en día. En este sentido, estos mismos autores señalan que la proximidad tiende, a menudo, a identificarse con el territorio, el cual se asocia a unos rasgos culturales y a una identidad propia. Hasta tal punto es así que, en lo que se refiere a los productos informativos, suele predominar (o al menos convivir a un mismo nivel) el criterio de tipo geográfico sobre el criterio de tipo temático.

Monreal (2013) lo resume señalando que los medios de proximidad se encargan de informar no solo sobre el ámbito local, sino desde ese mismo ámbito local. Y es desde dicho ámbito local donde tratan, en palabras de Esteve y Fernández del Moral (2007, p. 75), sobre "las vivencias directas de los ciudadanos, sus aspiraciones, necesidades y experiencias cotidianas".

Como ocurre con todo concepto, la definición de lo próximo y lo local siempre resulta más compleja de lo que una aproximación inicial pueda parecer. De hecho, un primer obstáculo que salvar es el hecho de que a menudo, especialmente cuando se trata de realizar análisis dentro del sector de la comunicación, ambos términos se reproduzcan como sinónimos, puesto que se estima que aquello que está próximo corresponde al ámbito de lo local. No obstante, siendo conscientes de la velocidad a la que se han reducido los tiempos y las distancias durante los últimos cien años, se puede comprobar cómo de difusa puede convertirse esa asociación. Hoy en día la proximidad y lo local resultan difícilmente equiparables, puesto que no existe una regla que especifique a partir de qué punto algo próximo puede considerarse como local. Así, dependiendo del contexto, un evento local puede ser considerado como un evento de un municipio, de una comarca, o de toda una región (Monreal, 2013). Resulta paradigmático además que cuanto más extensa es la globalización, mayor fuerza e interés adquiere la información de proximidad. Así lo señalan López y Maciá (2007), resaltando que este tipo de información ha ido ganando espacio a la información generalista. Algo que López (2004,p. 121) explicaba señalando que en un mundo globalizado el usuario de los medios tiende a querer "reconocerse en la información, autoafirmarse e identificarse, para lo que encuentra un campo ideal en los medios de proximidad".

Para explicar el proceso de descentralización de los medios existentes, de forma más o menos idéntica en el tiempo para la mayor parte de Europa, además de las razones desde el punto de vista de tratamiento de la información y la labor de los comunicadores, también hay que tener presente otro tipo de motivaciones, como es el caso de la descentralización administrativa, el multilingüismo, la defensa de la cultura, además de las razones económicas y de negocio (Garitaonandía, López y de Moragas, 1999). Atendiendo a estos criterios, se puede comprobar que en el caso español se encuentran todos ellos, aunque bien es cierto que, como resaltan Azurmendi, Manfredi y López (2011), en el caso nacional el desarrollo de las diferentes televisoras de proximidad ha ido estrechamente ligado a la descentralización administrativa y política experimentada, estando entre sus objetivos principales la puesta en valor de las culturas y lenguas de cada una de las regiones. A nivel práctico, esto ha supuesto una clara preeminencia del género de informativos, convirtiéndose durante décadas en el ejemplo máximo de las televisoras de proximidad del país. No obstante, con los años esta característica se ha ido suavizando y ya no se centra exclusivamente en los tradicionales telediarios, abriendo paso a otros géneros como son los reportajes, las tertulias, los magazines, etc. 
Dicha tendencia inicial fue resultado de la herencia que supuso el que las grandes cadenas de televisión nacionales abrieran franjas de información local o regional, un aspecto este que ocurrió de forma similar a lo largo de Europa. No obstante, lo que caracterizaba a la información que se proveía desde estas cadenas centralizadas, al contrario de lo que ocurriría con la aparición de los medios de proximidad, era que se tratase de información escasa, muy esquematizada y a la que se destinaban pocos medios. Y es que la aparición de los medios locales y regionales supuso que entre emisores y receptores se diera una coexistencia en un mismo contexto, lo que suponía la clave para que el tratamiento de la información adquiriese el nivel requerido para informar sobre la proximidad (Garitaonandía, López y de Moragas, 1999).

Con la aparición de las nuevas tecnologías, fundamentalmente con internet y su difusión a prácticamente cualquier dispositivo electrónico, los medios de comunicación tradicionales han sufrido un fuerte impacto a todos los niveles, desde su seguimiento hasta su capacidad económica o de negocio. Esto ha ocurrido también con la televisión por internet. Como señala Álvarez (2011), los cambios sociales en este sentido han sido determinantes y la televisión que está surgiendo se corresponde con una nueva sociedad puesto que la red de redes es incluso más trascendente que la aparición de la televisión de masas en el siglo pasado. No obstante, de acuerdo con lo señalado por Puntí (2014), tras una reestructuración y adaptación a los tiempos, el impacto económico se logrará superar, adecuándose un espacio suficiente para los diferentes soportes. Sin embargo, hay que incidir en la idea de que la función esencial de estos medios no puede ser olvidada, teniendo que seguir siendo el punto de unión y diálogo en una construcción social más justa y participativa. Es más, en el caso de los medios de proximidad, dentro del fenómeno globalizador que todo lo engloba, su función resulta vital en los procesos de generación de identidad y comunidad. Y es que como también aduce Puntí (2014), a pesar de que surjan fórmulas novedosas de gestión de la información, las materias primas no dejarán de ser las mismas. Así, siguiendo a Giddens (1995), son los medios de proximidad los que pueden garantizar la permanencia y el enraizamiento de lo particular, sirviendo al mismo tiempo de elemento identitario. Además, así como el conocerse, el identificarse, es un aspecto fundamental para consolidar el sentimiento de pertenencia, también lo es el darse a conocer, y de nuevo son los medios de proximidad los que tienen una posición ideal para ello. No obstante, ¿̇es esto lo que está haciendo Castilla-La Mancha Media?

\section{Metodología}

Uno de los objetivos marcados en el presente estudio es establecer hasta qué punto la programación de CMM (Castilla-La Mancha Media) contribuye a la construcción y promoción de la identidad castellanomanchega. Para analizar la programación de la Televisión de Castilla-La Mancha, siguiendo la línea marcada por Ruiz (2007), se han seleccionado una serie de indicadores que puedan aplicarse sobre los programas emitidos por el canal, tratando, de esta manera, de establecer si la programación ha estado planteada como una contribución a la promoción y difusión de los aspectos identitarios de la región. En total, se han establecido nueve grandes variables a tener en cuenta.

Lengua/Habla. El manchego como dialecto está incluido en la variedad castellana general, siendo la norma culta de esta la que sirve de guía para los usos de los hablantes de este territorio. El contexto sociocultural de la población dedicada a las actividades agrícolas y las tradicionalmente deficientes comunicaciones intrarregionales explican en Castilla-La Mancha la supervivencia de una serie de rasgos lingüísticos peculiares de carácter popular, claramente diferenciados del resto de la comunidad autónoma y del mundo hispánico. Este dialecto presenta una clara diferenciación a nivel fónico, encontrándose palabras como midiana, hurmiga, o almenaque que muestran la inestabilidad de las vocales átonas, lo que a su vez propicia la apertura de ei en ai (de manera que en lugar de afeitarse es común decir afaitarse), la formación de hiatos donde no deberían existir (en lugar de barajar se dice barajear). Otro rasgo distintivo es la aspiración de la $s$, de manera que en el manchego no se 
pronuncia asco como [ 'as ko ], sino ahco (Hernando, 2009). En el caso del estudio que nos ocupa, trataremos de observar cómo de presente se haya el dialecto manchego en la programación de CMM.

Historia. Como señala la misma página oficial de la Comunidad Autónoma, Castilla-La Mancha es una región joven con siglos de historia. Se trata de una región con restos ibéricos y celtibéricos, así como también romanos y arábigos. Además, la conquista cristiana tiene también una gran relevancia en la historia de la región, dejando notables huellas a lo largo y ancho de la región. Durante el resto de la Edad Media y la Edad Moderna las principales ciudades de la Comunidad vieron ampliada su importancia, aunque durante buena parte de la Edad Contemporánea la tónica característica fue la emigración hacia otros puntos peninsulares. Sin embargo, en tiempos más presentes, se ha conseguido invertir esa tendencia y, actualmente, la Comunidad ha pasado a ser receptora de población, es decir el saldo migratorio ya no es negativo sino positivo, un aspecto este que tiene que ver con la historia reciente de la Comunidad, pasando a ser una de las referencias en lo que se refiere a la vida rural (Castilla-La Mancha, 2018). Por tanto, el objetivo será determinar hasta qué punto se hace una representación y promoción de la historia de Castilla-La Mancha en la programación del canal de televisión, bien sea en documentales, noticiarios, programas especializados, series o incluso películas.

Literatura/Artes. Hablar de literatura representativa de Castilla-La Mancha significa, ineludiblemente, hablar de Cervantes y, sobre todo, de El Ingenioso Hidalgo Don Quijote de La Mancha. No en vano, las cifras que se manejan actualmente en torno a la obra más representativa del Siglo de Oro español indican que se han vendido más de 400 millones de copias (Información, 2016) y se ha traducido, durante el siglo XX y XXI, a 102 lenguas (Muñoz, 2016). No obstante, y como no puede ser de otra manera, son muchos las autoras y autores de mucha más actualidad a los que también se debe hacer referencia. En cuanto al resto de las artes, son muchos los ejemplos que se podrían destacar, caso de la Catedral de Toledo, las Casas Colgadas de Cuenca, el arte íbero de la Necrópolis de los Villares (Albacete), el Palacio del Infantado en Guadalajara, o la iglesia de San Pedro y San Pablo de Ciudad Real, por poner algunos ejemplos. Además, del adoptado y mundialmente conocido artista Doménikos Theotokópoulos "El Greco", que habiendo vivido 37 años en diferentes puntos de la geografía castellanomanchega, bien puede ser considerado como un referente del arte de la Comunidad. En este sentido, y como en los casos anteriores, se tratará de observar la relevancia y prevalencia que la programación de CastillaLa Mancha Media otorga a este aspecto de la cultura de Castilla-La Mancha.

Fiestas/costumbres/folclore. Si hay algo de lo que todo pueblo y región se siente orgulloso es de sus fiestas y costumbres. En este caso, existen tantos parecidos como matices, de manera que cada pueblo tiene sus peculiaridades y detalles. Unas fiestas y costumbres que son, sin lugar a dudas, el producto de una herencia cultural, las raíces históricas de todo pueblo que sirven de reconocimiento e integración, de unión e identificación de sus habitantes en torno a una forma de celebrar y ver la vida. Es por ello, que de nuevo nos hallamos ante una de las características que debería ser reflejada y promovida por la televisión autonómica. Además, no hay que olvidar el aspecto musical de las festividades y celebraciones en general, por lo que el folclore musical también debería tener su espacio representativo en la programación de la cadena.

Características socioeconómicas. Una de las cosas que más representan a Castilla-La Mancha, es la ruralidad y el mantenimiento de oficios tradicionales. Es por ello que se entiende que, siendo elementos tan distintivos y propios, la programación de Castilla-La Mancha Media debería ser una ventana que refleje dicha realidad.

Deportes. Uno de los aspectos que más pasiones levanta y más identificaciones consigue hoy en día es el deporte, y sobre todo el llamado "deporte rey", el fútbol, que es un elemento indisociable en lo que se refiere al sentido de pertenencia. Aunque también existen otros deportes como el balonmano, el futbol sala, que también gozan de buena salud en la comunidad. Es por ello que debería formar parte de la parrilla televisiva, así como de los diferentes noticiarios que se ofrezcan. Además, también sería recomendable, que más a nivel divulgativo y quizás como parte de la representación de costumbres y fiestas, se hiciera mención a juegos tradicionales. 
Simbología. Con esta variable, la intención es observar la prevalencia de la bandera y de las cinco provincias castellanomanchegas.

Paisaje. Se pretende determinar hasta qué punto los diferentes paisajes que componen la Comunidad forman parte de la programación de la televisión. Así, se espera que existan documentales, series e incluso películas que tengan los diferentes escenarios de Castilla-La Mancha como contexto principal.

Gastronomía. Finalmente, un aspecto que no podía descuidarse es la presencia en Castilla-La Mancha Media de la gastronomía típica de la región. Una presencia que podría estar tanto en programas especializados de cocina, como en los noticiarios a la hora de cubrir eventos festivos, o incluso las posibles series o películas que tengan su discurso basado en la Comunidad.

En base a estos nueve indicadores, se ha realizado un análisis de la parrilla de programación de Castilla-La Mancha Media, diferenciando la programación habitual de los días de entre semana (de lunes a jueves) y la programación especial de fin de semana (empezando por el viernes). Hay que señalar que se ha respetado la tipología otorgada a cada espacio por la misma cadena, de manera que se puede entender mejor la concepción que de estos se tiene desde producción y dirección. Conviene resaltar también que se ha hecho uso de la programación vigente en el momento en que se redactaba esta investigación, pero solo cuando, al compararla con el histórico, las tipologías de las emisiones coincidían en su mayor medida (puesto que los programas que se emiten van variando con el tiempo); en este sentido la parrilla sufre más modificaciones durante el fin de semana por lo que se ha optado por no indicar el nombre del programa en los casos en que las tipologías se intercambiasen dependiendo de la hora o la época del año. Para facilitar el análisis, en cada una de las tipologías se muestra un cuadro en el que aparecen las variables analizadas y una puntuación del 1 al 5, siendo 1 el nivel de representación más bajo y 5 el nivel de representación más elevado. La recogida de datos para este análisis se ha basado en el contenido disponible en el apartado "a la carta" de la página web oficial de CMM, así como siguiendo la programación histórica de canal, especialmente para comprobar el tipo de películas programadas.

\section{Resultados y discusión}

Tras realizar una revisión de las películas programadas a lo largo de los años por la televisión castellanomanchega, en la tabla 1, se ha observado que se trata de películas que corresponden al cine americano de segunda fila, westerns, y cine español de los años 70 y 80 del siglo XX (básicamente comedia ligera y vodevil costumbrista). Esto significa que la representación castellanomanchega en las películas que se programan es cercana a cero (no se trata de un cero absoluto, puesto que algunas de las películas españolas son susceptibles de mostrar alguno de los aspectos identitarios castellanomanchegos indicados en este estudio, aunque se trata de una representación anecdótica). No obstante, resulta entendible considerando que no existe una industria del cine en la región, de manera que resulta poco viable el poder conseguir que dicha representación sea significativa. 
Tabla 1

Análisis de representación castellanomanchega en el cine a partir de los indicadores identitarios

\begin{tabular}{|lllllll|}
\hline Lengua/habla & 1 & 2 & 3 & 4 & 5 & Media \\
Historia & $\#$ & & & & & \\
Literatura/Artes & $\#$ & & & & & \\
Fiestas/Costumbres & $\#$ & & & & & \\
C. Socioeconómicas & $\#$ & & & & \\
Deportes & $\#$ & & & & \\
Simbología & $\#$ & & & & \\
Paisaje/Patrimonio & $\#$ & & & & \\
Gastronomía & $\#$ & & & & \\
\hline
\end{tabular}

Fuente: elaboración propia

Por lo que se refiere a deportes, tabla 2, CMM dispone de varios espacios deportivos dedicados a cubrir la información y retransmisiones de las diferentes competiciones en las que existe representación por parte de equipos de Castilla-La Mancha, tales como balonmano, fútbol y fútbol sala, tanto en equipos seniors de ambos sexos, como en equipos juveniles e infantiles. Asimismo, también se retransmite cualquier otra modalidad deportiva y/o acontecimiento de relevancia deportiva que tenga lugar en la Comunidad, como puede ser el Máster Nacional de Bádminton sub-19, campeonatos de motociclismo, atletismo, etc. Estando disponibles, en su mayoría, en la plataforma digital CMMPLAY. Dicha cobertura se realiza a través del programa televisivo "La cancha regional" y el radiofónico "Castilla-La Mancha en juego". En "La cancha regional", buena parte de las retransmisiones y o resúmenes se ofrecen sin ningún tipo de comentario o comentarista, mostrándose tan solo las imágenes y el sonido en vivo (o en su defecto canciones de fondo).

En otras ocasiones, cuando se trata de eventos de más empaque, sí que se dispone de uno o dos comentaristas locales, con lo que se hacen referencias puntuales al tipo de habla típico de la zona, aunque sin ser un espacio referente para estos fines. En este sentido, se tiene como director del área de deportes al toledano Alberto Jiménez, quien ha conformado el equipo de deportes de la cadena con Álvaro Seguí, David Vidales, Aurora González y Alberto Abril.

Tabla 2

Análisis de representación castellanomanchega en los prog, deportivos, a partir de los indicadores identitarios

\begin{tabular}{|c|c|c|c|c|c|c|}
\hline & 1 & 2 & 3 & 4 & 5 & Media \\
\hline Lengua/habla & & \# & & & & \\
\hline Historia & & & & & & \\
\hline Literatura/Artes & & & & & & \\
\hline Fiestas/Costumbres & & & & & & \\
\hline C. Socioeconómicas & & & & & & 0,77 \\
\hline Deportes & & & & & $\#$ & \\
\hline Simbología & & & & & & \\
\hline Paisaje/Patrimonio & & & & & & \\
\hline Gastronomía & & & & & & \\
\hline
\end{tabular}

Fuente: elaboración propia

La sección de documentales, en la tabla 3, se dedica en su mayor parte a la revisión y difusión de los diferentes contextos que se pueden hallar en Castilla-La Mancha, siendo el campo, la ganadería y el mundo rural en general, 
el principal tema que se trata. Así, el canal tiene programas como "Mundo Rural" que por un espacio de unos 30 minutos tratan una amplia diversidad de temas relacionados con dicho mundo rural. Este tipo de espacios se está aprovechando de forma fantástica para difundir muchas de las características propias de la Comunidad, tanto a nivel de habla, costumbres, características socioeconómicas, paisaje, historia, etc.

Tabla 3

Análisis de representación castellanomanchega en los documentales a partir de los indicadores identitarios

\begin{tabular}{|c|c|c|c|c|c|c|}
\hline & 1 & 2 & 3 & 4 & 5 & Media \\
\hline Lengua/habla & & & & & \# & \\
\hline Historia & & & & & $\#$ & \\
\hline Literatura/Artes & & & & \# & & \\
\hline Fiestas/Costumbres & & & & \# & & \\
\hline C. Socioeconómicas & & & & & \# & 3,3 \\
\hline \multicolumn{7}{|l|}{ Deportes } \\
\hline \multicolumn{7}{|l|}{ Simbología } \\
\hline Paisaje/Patrimonio & & & & & \# & \\
\hline Gastronomía & & \# & & & & \\
\hline
\end{tabular}

Fuente: elaboración propia

Dentro de los programas de entretenimiento, tabla 4, se halla una gran variedad, existiendo programas de carácter romántico como "¿Te quieres casar conmigo?"; musicales como "A tu vera" y "A tu vera Mini"; programas como "Ancha es Castilla-La Mancha”, "Aquí no hay playa ¿Y qué?”, "El cuentakilómetros", o "CastillaLa Mancha Me Gusta", que muestran las costumbres pasadas y actuales de los castellanomanchegos; programas con historias personales de gente desconocida de la Comunidad, caso de "Gente Maravillosa" o "Héroes anónimos", mientras que "Este es mi sitio" muestra las historias de gente más reconocible de la región; finalmente, también hay que destacar la existencia de un programa de variedades como es "Estando contigo" o el programa estrella de la cadena, "En Compañía", presentado por el popular presentador Ramón García. En todos ellos se muestra a personas locales, de manera que el habla castellanomanchega está ampliamente representada, asimismo también se hace una referencia directa a fiestas y costumbres, características socioeconómicas, paisaje y patrimonio, así como también a la gastronomía.

Tabla 4

Análisis de representación castellanomanchega en los prog. de entretenimiento a partir de los indicadores

\begin{tabular}{|c|c|c|c|c|c|c|}
\hline & 1 & 2 & 3 & 4 & 5 & Media \\
\hline Lengua/habla & & & & & $\#$ & \\
\hline Historia & \# & & & & & \\
\hline Literatura/Artes & $\#$ & & & & & \\
\hline Fiestas/Costumbres & & & & & $\#$ & \\
\hline C. Socioeconómicas & & & & & $\#$ & 2,8 \\
\hline \multicolumn{7}{|l|}{ Deportes } \\
\hline \multicolumn{7}{|l|}{ Simbología } \\
\hline Paisaje/Patrimonio & & & & & $\#$ & \\
\hline Gastronomía & & & & \# & & \\
\hline
\end{tabular}

Fuente: elaboración propia

Por lo que se refiere a los espacios informativos, tabla 5, CMM emite cuatro espacios diferenciados: 3 informativos generales ( $8 \mathrm{am}, 2 \mathrm{pm}$, y $8 \mathrm{pm}$ ), 2 informativos especiales sobre el campo (a medio día y por la 
noche), 2 informativos especiales sobre deporte (a medio día y por la noche), y 2 informativos especiales sobre el tiempo meteorológico (también a medio día y por la noche), además de la repetición de madrugada del pack informativo de la noche anterior. Estos informativos tienen un carácter generalista, prestando atención a la información nacional e internacional con cierto alcance sobre la región, pero poniendo el foco en la actualidad regional.

En cuanto a los indicadores identitarios, los programas informativos son susceptibles de evidenciarlos todos en mayor o menor medida, por lo que se ha decidido establecer una valoración media para todos ellos, a excepción del de lengua/habla, puesto que este es un valor con una representación mucho mayor, tanto por su uso por parte de los corresponsales como, sobre todo, por su uso por parte de la gente local entrevistada.

\section{Tabla 5}

Análisis de representación castellanomanchega en los informativos a partir de los indicadores identitarios

\begin{tabular}{|c|c|c|c|c|c|c|}
\hline & 1 & 2 & 3 & 4 & 5 & Media \\
\hline Lengua/habla & & & & & \# & \\
\hline Historia & & & $\#$ & & & \\
\hline Literatura/Artes & & & \# & & & \\
\hline Fiestas/Costumbres & & & \# & & & \\
\hline C. Socioeconómicas & & & \# & & & 3,2 \\
\hline Deportes & & & \# & & & \\
\hline Simbología & & & $\#$ & & & \\
\hline Paisaje/Patrimonio & & & \# & & & \\
\hline Gastronomía & & & \# & & & \\
\hline
\end{tabular}

Fuente: elaboración propia

Por lo que se refiere a series, tabla 6, durante el último año CMM ha programado tres: "La esclava blanca" (telenovela colombiana), "La voz del arrabal" (telenovela colombiana), y "Una aldea francesa" (serie francesa). Ninguna de las tres tiene carácter local o regional, sino que se trata de series extranjeras adquiridas a través de FORTA, federación de la que la cadena forma parte. Se trata, sin duda, de una ocasión perdida para incrementar el objetivo y fomentar las características socioculturales de la región castellanomanchega, papel que, como se ha podido comprobar en apartados anteriores, se le supone a una televisión de proximidad. Sin embargo, se deduce que el considerable gasto que supone la producción de un producto televisivo de estas características ha hecho poco posible su realización.

Tabla 6

Análisis de representación castellanomanchega en las series a partir de los indicadores identitarios

\begin{tabular}{|c|c|c|c|c|c|c|}
\hline & 1 & 2 & 3 & 4 & 5 & Media \\
\hline \multicolumn{7}{|l|}{ Lengua/habla } \\
\hline \multicolumn{7}{|l|}{ Historia } \\
\hline \multicolumn{7}{|l|}{ Literatura/Artes } \\
\hline \multicolumn{7}{|l|}{ Fiestas/Costumbres } \\
\hline C. Socioeconómicas & & & & & & 0 \\
\hline \multicolumn{7}{|l|}{ Deportes } \\
\hline \multicolumn{7}{|l|}{ Simbología } \\
\hline \multicolumn{7}{|l|}{ Paisaje/Patrimonio } \\
\hline Gastronomía & & & & & & \\
\hline
\end{tabular}

Fuente: elaboración propia 
Uno de los aspectos a destacar en la programación de CMM son los programas de tipo taurino, así como la retransmisión de corridas de toros. Con José Miguel Martín de Blas al frente, el canal, con "Tiempo de Toros", hace un repaso semanal de las novedades dentro del mundo taurino o la retrasmisión de los festejos que se estén llevando a cabo tanto a nivel regional como a nivel nacional. Debido a lo especializado de la temática, y tal y como se puede observar en la tabla 7, son pocos los indicadores identitarios que en este tipo de programas se representan.

Tabla 7

Análisis de representación castellanomanchega en los progr. taurinos a partir de los indicadores identitarios

\begin{tabular}{|c|c|c|c|c|c|c|}
\hline & 1 & 2 & 3 & 4 & 5 & Media \\
\hline Lengua/habla & \# & & & & & \\
\hline Historia & $\#$ & & & & & \\
\hline \multicolumn{7}{|l|}{ Literatura/Artes } \\
\hline Fiestas/Costumbres & & & & & $\#$ & \\
\hline C. Socioeconómicas & & & & & & 0,88 \\
\hline \multicolumn{7}{|l|}{ Deportes } \\
\hline \multicolumn{7}{|l|}{ Simbología } \\
\hline Paisaje/Patrimonio & $\#$ & & & & & \\
\hline Gastronomía & & & & & & \\
\hline
\end{tabular}

Fuente: elaboración propia

Poniendo en contraste los resultados obtenidos al analizar los indicadores identitarios encontrados en los programas emitidos por el canal televisivo castellanomanchego, se ha obtenido una media de 1,76 sobre 5 . La máxima puntuación la han obtenido los programas documentales $(3,3)$ y los informativos $(3,2)$, mientras que las series $(0)$, los deportes $(0,77)$ los toros $(0,88)$ son el tipo de programa que un menor porcentaje de indicadores identitarios han registrado, no obstante, se trata de unos datos que necesitan de matización, puesto que, tanto por arriba como por abajo, pueden resultar engañosos.

En el caso de valoración alta, los documentales han sido los que han obtenido mayor puntuación en cuanto a representación de los indicadores identitarios, por lo que se podría pensar que son el tipo de programa que más valor aportan a la cadena en cuanto a lo que se refiere a la difusión de las características de lo castellanomanchego. Sin embargo, como se vio anteriormente, estos documentales se programan antes de las $8 \mathrm{am}$, lo que supone estar en pantalla en una franja horaria de poca audiencia, de manera que se puede esperar que tenga un impacto menor sobre los espectadores. No obstante, es cierto que el canal permite la visualización de este tipo de programas de manera online mediante la plataforma CMMPLAY, lo que podría hacer pensar que la exposición al público podría ser mayor a lo esperado atendiendo solo a la hora de programación televisiva. En cambio, teniendo en cuenta que en Castilla-La Mancha la población mayor a los 50 años supone casi el 38\% de la población (INE, 2019), significando una menor tasa de acceso a los contenidos en internet y, sin embargo, siendo los que mayor número de horas ven la televisión (4 horas y 44 minutos de media para la población de entre 45 y 64 años, y 6 horas y 4 minutos para los mayores de 65 años), se puede aceptar la idea de que la posibilidad de encontrar los documentales en la plataforma online no va a suponer una mayor visualización por parte de estos grupos de edad.

Por lo que se refiere a los programas de deportes y de toros, si bien es cierto que las valoraciones obtenidas son bajas, esto se explica por lo especializado de su contenido. Es decir, se trata de dos temáticas muy arraigadas (aunque bien es cierto que la afición taurina está decayendo con fuerza, pasando de 575 festejos taurinos celebrados en Castilla-La Mancha en 2010 (Ministerio de Educación, Cultura y Deporte, 2012), a 347 en el año 2018 (Ministerio de Educación, Cultura y Deporte, 2019), en la cultura popular de la región. Por lo tanto, serían 
dos emisiones que se podrían considerar como participantes de la difusión de las características socioculturales de la Comunidad.

\section{Conclusiones}

Se puede considerar que, en general, la programación del canal cumple con lo esperado para una televisión de proximidad, puesto que, a pesar de que se ha obtenido una media de 1,76 sobre 5 en el análisis realizado, se ha comprobado que dicha puntuación se ha visto muy negativamente afectada por el hecho de que no existan series propias (probablemente debido al reducido presupuesto de que se dispone, y a la escasez de productoras locales capaces de llevar a cabo un proyecto de dicha envergadura), y a la existencia de dos tipologías de programas muy específicas (deportes y toros) que difícilmente pueden cumplir con muchas de las variables establecidas para el estudio. Tanto es así que, si se obvian dichas tipologías, el resto de la programación obtiene una puntuación de 2,5 sobre 5 (teniendo en cuenta el cine, en el cual hemos visto tiene un problema similar al de las series, aunque ligeramente subsanado por el hecho de programar películas españolas que son susceptibles de, en algún momento, hacer referencia a la región estudiada), o, incluso no teniendo en cuenta tampoco el cine, una puntuación de 3,1 sobre 5 en lo que a la presencia de indicadores identitarios en la programación CMM se refiere. Podemos señalar, por tanto, que la programación de Castilla-La Mancha Media tiene un grado medio de identidad colectiva, que se visualiza sobre todo en los programas de "entretenimiento" (2,8 sobre 5), destacando la falta de símbolos que aporten una clara identidad castellanomanchega y una falta de aspectos identitarios relacionados en el ámbito cultural y formativo en sus contenidos.

La propuesta de indicadores de identidad que se ha realizado se revela útil para detectar las debilidades y las fortalezas identitarias en los programas de televisión regional, pero se ha detectado una gran dificultad para señalar los aspectos o elementos que actúan en la construcción de la identidad cultural de una población. Hay que tener en cuenta que uno de los objetivos de los medios de comunicación de proximidad es precisamente la divulgación y promoción de la identidad cultural del territorio donde ejerce su actividad y parece razonable que haya una herramienta que mida el cumplimiento de dicho objetivo. En el caso castellanomanchego esta dificultad de la que hablamos es aún mayor ya que cuando se trata de la identidad y la cultura de esta comunidad, un $76 \%$ considera la castellanomanchega y española, que o no tienen ninguna diferencia o de tenerla es escasa salvo algún rasgo distintivo. Este es uno de los datos relevantes que hemos obtenido del estudio de opinión realizado entre los ciudadanos castellanomanchegos y que forma parte de la investigación completa a la que pertenece este artículo. La identidad propia de la autonomía se diluye dentro de la genérica española, sin que la mayoría de las personas vea características particulares que se diferencien fácilmente.

Otros aspecto destacable tras los resultados obtenidos, es valorar la misión que deben de tener las televisiones de proximidad en la sociedad española del siglo XXI, su rentabilidad económica y social dentro del fenómeno globalizador que nos envuelve a todos, si pueden garantizar la permanencia de lo particular y sirven de elemento identitario para consolidar el sentimiento de pertenencia, ya que los modelos con los que nacieron este tipo de televisoras se han quedado obsoletos y en la mayor parte de los casos están claramente sobredimensionados al ver el uso que se hace de los mismos. En el caso de Castilla-La Mancha, los ciudadanos opinan en su mayoría, que la televisión regional cumple un rol importante para su territorio y que es necesario que exista esa información de proximidad, aunque en realidad la tendencia global de la cadena ha sido, desde 2005, a disminuir de una forma más bien constante su índice de audiencia, especialmente a partir de la segunda década del siglo XXI.

Por lo que se refiere a la comparación con el resto de televisiones de carácter regional, CMM se sitúa en la séptima posición, siendo la cuarta de entre los canales cuya comunidad no tiene una lengua propia diferente a 
la del castellano, por detrás de Canal Sur, Aragón TV y Extremadura TV; y situándose por delante de TPA (Asturias), TVCan (Canarias) o Telemadrid.

Ante este panorama, no se puede más que remitirse a las conclusiones que se alcanzan desde Barlovento (2019), coincidiendo con ellos al señalar que, si bien las televisoras autonómicas consiguieron estabilizar e incluso incrementar en algunos casos la fidelidad de las audiencias, "más temprano que tarde las cadenas autonómicas también deberán reflexionar y poner en marcha un nuevo modelo de negocio, pues como todos conocemos hay declaraciones políticas amenazadoras de su viabilidad futura".

\section{Referencias bibliográficas}

ÁLVAREZ, Abelardo. (2016): Cultura e identidad frente a la globalización en La cultura en función del trabajo comunitario. Barcelona (España): Editorial Académica Española.

ÁLVAREZ, José. (2011). La televisión etiquetada: nuevas audiencias, nuevos negocios. Fundación Telefónica.

AZURMENDI, A.; MANFREDI, J. L. y LÓPEZ, N. (2011): La reforma de las televisiones autonómicas a partir de la nueva regulación audiovisual, Ley 7/2010 General de la Comunicación Audiovisual. Derecom.com. Recuperado de: http://www.derecom.com/component/k2/item/141-la-reforma-de-la-television-publicaautonomica-en-el-nuevo-marco-legal-audiovisual-ley-7-2010-general-de-la-comunicacion-audiovisual-decobertura-nacional

BARLOVENTO (2019). Análisis Televisivo 2018. Barlovento Comunicación. Recuperado de: https://www.barloventocomunicacion.es/wp-content/uploads/2018/12/analisis-televisivo-2018BarloventoComunicacion.pdf

CASTELLS, Manuel. (2003). La Era de la información: economía, sociedad y cultura (Volumen 2). Alianza Editorial.

CASTILLA-LA MANCHA (2018). Una región joven con siglos de historia. Recuperado de:

https://www.castillalamancha.es/clm/historia/siglosdehistoria

CMM (2019 a). Programación TV. Castilla-La Mancha Media. Recuperado de: https://www.cmmedia.es/programacion/tv/

ESTEVE, Francisco, y FERNÁNDEZ, Javier. (2007). Áreas de Especialización Periodística. Madrid: Fragua.

GARITAONANDÍA, Carmelo; LÓPEZ, Bernat; y DE MORAGAS, Miguel. (Eds.). (1999). Televisión de proximidad en Europa: experiencias de descentralización en la era digital. Universitat Autònoma de Barcelona. Servei de Publicacions.

GIDDENS, Anthony. (1995). La constitución de la sociedad: bases para la teoría de la estructuración. Buenos Aires: Amorrortu

GIMÉNEZ, Gilberto. (2005). La cultura como identidad y la identidad como cultura. Consejo Nacional de la Cultura y las Artes. México. Recuperado de: https://www.academia.edu/download/35120936/7_LA_CULTURA_COMO_IDENTIDAD_Y_LA_IDENTIDAD_COMO_CULTURA.pdf

GONZÁLEZ, Ignacio. (2018). Conservación de bienes culturales: teoría, historia, principios y normas. Cátedra.

HALL, Stuart. (1996). Introduction: Who Needs 'Identity'? En HALL, Stuart., \& DU 
HERNANDO, Luis Alberto (2009). Sobre el habla regional de La Mancha. Revista de Filología Románica, 26, 171186.

INE (2019). Población residente por fecha, sexo y edad y por Comunidades Autónomas. Instituto Nacional de Estadística. Recuperado de: https://www.ine.es/jaxiT3/Datos.htm?t=9681\#!tabs-tabla

INFORMACIÓN (2016). Las cifras de Miguel de Cervantes. Diario Información. Recuperado de: https://www.informacion.es/cultura/2016/04/22/cifras-miguel-cervantes-6187907.html

LÓPEZ, Xosé (2004). Desafíos de la comunicación local: guía para la práctica de la información en los ámbitos de proximidad. Comunicación Social.

LÓPEZ, Xosé, y MACIÁ, Juan. (2007). Periodismo de proximidad. Madrid: Síntesis.

MINISTERIO DE EDUCACIÓN, CULTURA Y DEPORTE (2012). Estadística de Asuntos Taurinos 2007 - 2011. Ministerio de Educación, Cultura y Deporte. Recuperado de: http://www.culturaydeporte.gob.es/dam/jcr:c5822e5a-d7b4-47ee-b0f762e2e1eca37c/Estadistica_de_Asuntos_Taurinos_2007-2011_Sintesis_de_Resultados.pdf

MINISTERIO DE EDUCACIÓN, CULTURA Y DEPORTE (2012). Estadística de Asuntos Taurinos 2012 - 2018. Ministerio de Educación, Cultura y Deporte. Recuperado de: http://www.culturaydeporte.gob.es/dam/jcr:5b65492f-a60a-4168-82a9-04fe9ee46205/estadistica-deasuntos-taurinos-2012-2018.pdf

MOLANO, Olga. L. (2007). Identidad cultural un concepto que evoluciona. Opera, 7(7), 69-84. Recuperado de: https://www.metarevistas.org/index.php/opera/article/viewFile/1187/1126

MONREAL, Julio. (2013). Periodismo local. En Cebrián, B. y Mirón. L.M. (coord.). Áreas del periodismo. Salamanca: Comunicación Social.

MUÑOZ, Rafael. (2016). Una aproximación a la difusión del Quijote a través de sus ediciones. eXtoikos, (1), 5964. Recuperado de: https://dialnet.unirioja.es/descarga/articulo/5719672.pdf

PUNTÍ, Mónica (2014): Estudio del caso de Nació Digital como medio digital de proximidad. En SABÉS, Fernando, y VERÓN, José Juan (Coord.). Universidad, Investigación y periodismo digital. Asociación de periodistas de Aragón.

RUÍZ, Antonia. (2007). Los instrumentos de medida de las identidades en los estudios del CIS y el Eurobarómetro: problemas de validez de la denominada escala Moreno. Revista Española de Investigaciones Sociológicas (REIS), 117(1), 161-182.

SAMOUR, Héctor. (2013). Globalización, cultura e identidad. Repositorio Digital de Ciencia y Cultura de El Salvador. Recuperado de: http://redicces.org.sv/jspui/bitstream/10972/831/1/globalizaci\%C3\%B3n_cultura_identidad.pdf

TOMLINSON, John. (1999). Globalization and culture. University of Chicago Press.

TOURAINE, Alain. (1987). El regreso del actor. Editorial Eudeba: Buenos Aires.

WALLERSTEIN, Immanuel. (1989). Culture as the ideological battleground of the modern world-system. Hitotsubashi journal of social studies, 21(1), 5-22. Recuperado de: http://hermes-ir.lib.hitu.ac.jp/hermes/ir/re/8420/HJsoc0210100050.pdf

Esta obra está bajo una Licencia Creative Commons Attribución-NoCommercial 4.0 International 